Revue des patrimoines

$17 \mid 2011$

Les patrimoines de l'enseignement supérieur

\title{
Faculté des sciences de Bordeaux, René-André Coulon architecte
}

Franck Delorme

\section{OpenEdition}

Journals

Édition électronique

URL : http://journals.openedition.org/insitu/932

DOI : 10.4000/insitu.932

ISSN : 1630-7305

Éditeur

Ministère de la culture

Référence électronique

Franck Delorme, «Faculté des sciences de Bordeaux, René-André Coulon architecte », In Situ [En ligne] 17 | 2011, mis en ligne le 26 janvier 2012, consulté le 30 avril 2019. URL : http:// journals.openedition.org/insitu/932 ; DOI : 10.4000/insitu.932

Ce document a été généré automatiquement le 30 avril 2019

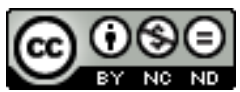

In Situ Revues des patrimoines est mis à disposition selon les termes de la licence Creative Commons Attribution - Pas d'Utilisation Commerciale - Pas de Modification 4.0 International. 


\title{
Faculté des sciences de Bordeaux, René- André Coulon architecte
}

\author{
Franck Delorme
}

En 1945, comme dans la plupart des grandes villes françaises, les bâtiments universitaires situés dans le centre ancien de Bordeaux sont au bord de l'asphyxie. Depuis 1900, les effectifs d'étudiants ont presque quadruplé, passant de 1800 à 6300 . Cela fait longtemps que les facultés aux architectures prestigieuses et monumentales édifiées place de la Victoire par Jean-Louis Pascal de 1876 à 1888 (médecine et pharmacie) et cours Pasteur par Charles Durand de 1880 à 1886 (sciences et lettres), ne remplissent plus correctement leur rôle ${ }^{1}$ (fig. $\left.\mathbf{n}^{\circ} \mathbf{1}\right)$. La faculté de médecine avait pu, en 1922, s'agrandir sur des terrains adjacents, « mais le nombre croissant des étudiants dans toutes les villes universitaires, la vétusté des anciens bâtiments, les rapides progrès de la science, exigèrent la création de nouveaux édifices et le rééquipement des anciens ${ }^{2}$.» 
Figure 1

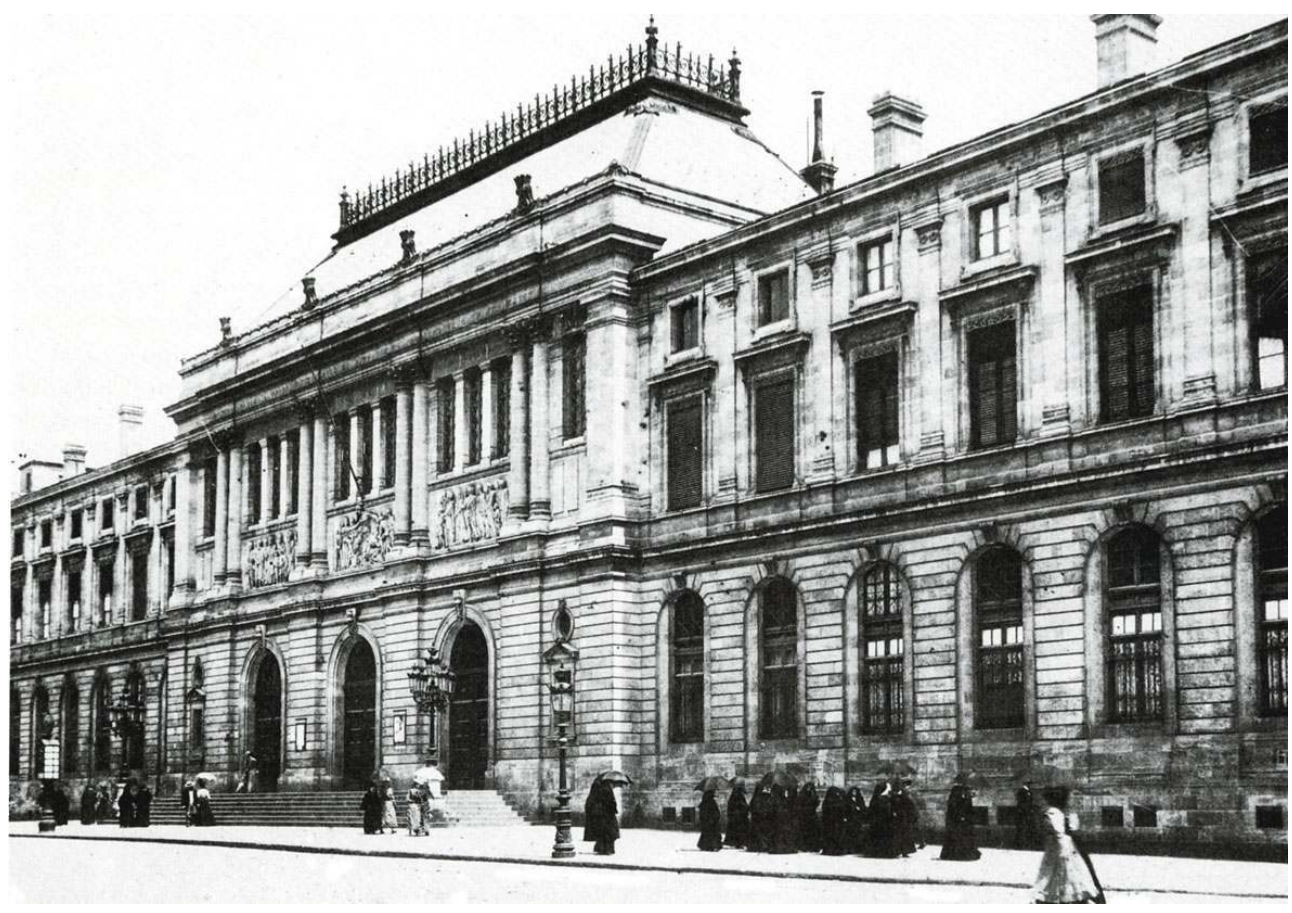

Façade de l'ancienne faculté des sciences sur le cours Pasteur, carte postale.

(c) Arch. Muni. de Bordeaux.

2 Au lendemain de la Seconde Guerre mondiale, deux solutions, aussi peu satisfaisantes et irréalistes l'une que l'autre, sont envisagées pour tenter de trouver de la place: l'aménagement de locaux existants en récupérant des bâtiments en plein centre de la ville ou l'acquisition de terrains pour permettre des constructions neuves. C'était se bercer de belles illusions. Profiter de bâtiments disponibles à court terme et à moindre frais, est aussi difficile que d'espérer bénéficier de réserves foncières en ville ou à proximité immédiate du centre.

\section{Problèmes de localisation}

Dès 1933, Roger-Henri Expert - alors architecte conseil de la ville - étudie plusieurs projets de déplacement de la faculté des sciences. Une possibilité d'implantation est envisagée cours de la Marne, non loin de la gare, sur des terrains appartenant à l'université de Bordeaux, sur une partie desquels se trouve déjà l'Institut de zoologie construit par l'architecte Ernest Lacombe de 1900 à $1903^{3}$.

4 La forme en sifflet et l'étroitesse du terrain imposent un jeu de contorsion pour faire rentrer au chausse-pied un programme assez consistant qui ambitionne de reloger l'intégralité des diverses disciplines scientifiques, tâche dont Expert se tire plutôt bien comme le montrent ses différents projets (fig. $\mathbf{n}^{\circ} \mathbf{2}$ ). Cette solution d'implantation est de nouveau brièvement évoquée après la guerre mais elle est vivement critiquée par Philippon, inspecteur départemental de l'urbanisme, comme un pis-aller ne servant qu'à reculer l'échéance à laquelle le déménagement des facultés du centre deviendra inévitable. 
Figure 2

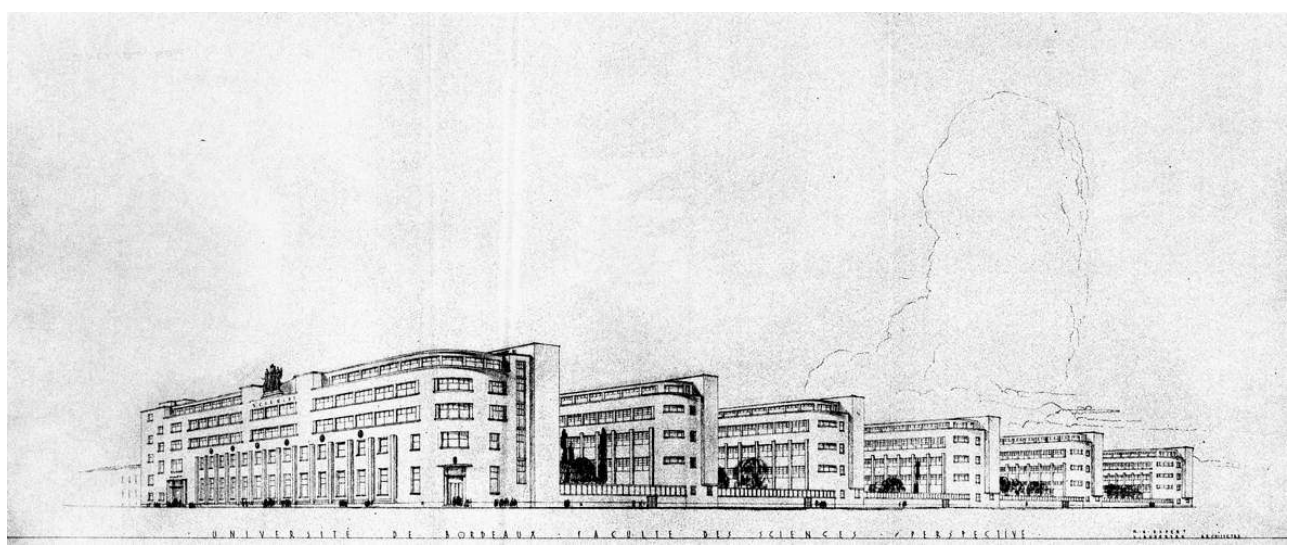

Perspective du projet de faculté des sciences cours de la Marne par Roger-Henri Expert en 1934.

(c) CNAM/SIAF/CAPA/Archives d'architecture du XXe siècle, fonds Expert 32 IFA.

En 1949, l'inspecteur départemental parvient à convaincre le maire de Bordeaux Jacques Chaban-Delmas et le doyen de l'université de la nécessité d'un achat conjoint de terrains en dehors de la ville, achat qui doit servir, dans l'esprit des autorités, d'amorce à un vaste et ambitieux projet de campus. La Ville se porte acquéreur, à Talence dans la proche banlieue sud-ouest de Bordeaux, du domaine du Castel-Terrefort, et l'université de celui du domaine du château Bonnefond. Lors de l'acquisition effectuée en 1951, l'ensemble des terrains totalise dix-neuf hectares qui sont destinés à la seule faculté des sciences. Plusieurs conditions sont à l'origine de cette courageuse prise de décision. «Au départ, pour la construction de la première tranche de la faculté des sciences (...), les crédits accordés sont importants. C'est une des premières universités françaises à quitter le centre ville. Les crédits sont assez larges et la volonté du doyen Brus de créer une université de prestige ont permis de construire un ensemble luxueux où chaque bâtiment est réservé à un seul enseignement ${ }^{4}$. »

L'opportunité de profiter d'importantes réserves foncières, mais à des prix abordables, est donc saisie. L'éloignement du centre est compensé par l'aspect vierge de l'emplacement qui laisse le champ libre à l'organisation urbaine et architecturale : «(...) le domaine universitaire se situera en limite de forêt, dans un secteur que les grands projets routiers de l'époque vouaient à l'abandon. Là, comme presque partout, les terrains achetés sont moins coûteux parce que peu attractifs: absence de voies de desserte secondaires, absence de transports en communs, absence de services ${ }^{5} . » ;$ au maître d'ouvrage et à l'architecte, la tâche de faire du site un ensemble universitaire complet et vivant.

\section{Les sciences mises au vert}

7 La période de la Reconstruction est propice aux programmes d'envergure et favorise les projets à forte qualité architecturale. Les constructions universitaires sont confiées à des architectes choisis au niveau de l'État sous l'autorité du ministre de l'Éducation nationale. Devant l'urgence de la situation bordelaise, la nomination de René-André Coulon (1908-1997) pour dresser le projet de déplacement de la faculté des sciences précède même la nomination de Louis Sainsaulieu comme architecte en chef de l'ensemble du 
domaine universitaire. Sainsaulieu, assisté du bordelais Pierre Mathieu, dressera en 1959 le plan de masse d'un véritable campus - approuvé en 1963 par le Conseil général des Bâtiments de France -, destiné à accueillir toutes les facultés (à l'exception de celles de médecine et de pharmacie qui rejoignent le CHU dans le secteur de Carreire, moins éloigné du centre) et également les instituts d'enseignement supérieur (fig. $n^{\circ} 3$ ).

Figure 3

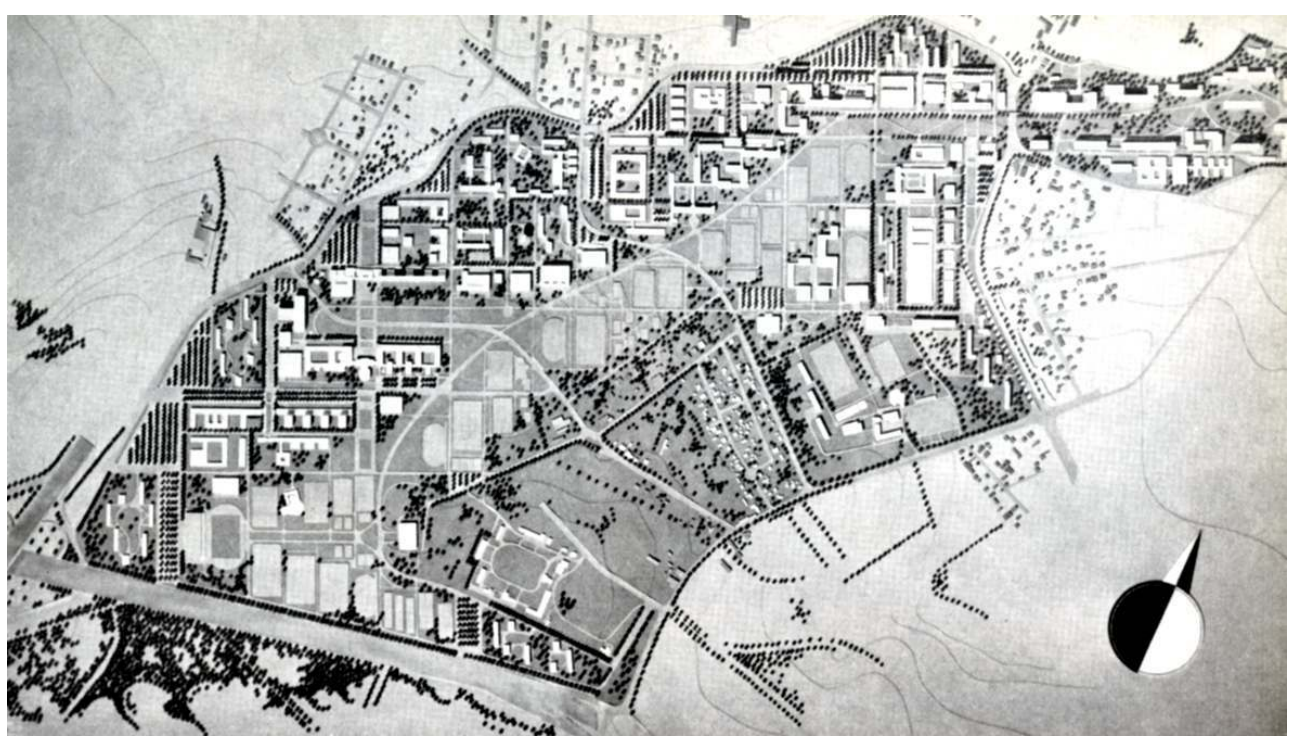

Plan du domaine universitaire de Bordeaux/Talence par Louis Sainsaulieu.

(c) CNAM/SIAF/CAPA/Archives d'architecture du XXe siècle, fonds DAU 133 IFA 225/1.

8 L'ensemble des Sciences conçu par Coulon constitue l'extrémité nord-est du futur domaine universitaire implanté sur les communes de Talence, de Pessac et de Gradignan, et en formera, en quelque sorte la porte d'entrée, voire d'honneur. «La qualité du site a permis de faire éclater l'implantation des constructions en spécialisant les bâtiments pour chaque discipline. C'est ainsi qu'il a été possible, tout en conservant le cadre naturel, de ménager entre les bâtiments des plages d'extension. Le secteur central est ceinturé par une circulation, pour voitures et pour piétons, qui dessert les entrées principales des bâtiments. À la périphérie, les entrées de service des bâtiments sont desservies par une voie qui borde un parking sur toute sa longueur ${ }^{6}$.» La situation actuelle montre que les prévisions et les précautions de l'époque étaient justifiées car les espaces libres ont été en grande partie construits et l'ensemble de la faculté s'est considérablement densifié, mais le site conserve encore beaucoup de qualités (fig. $\mathbf{n}^{\circ} \mathbf{4}$ ). 


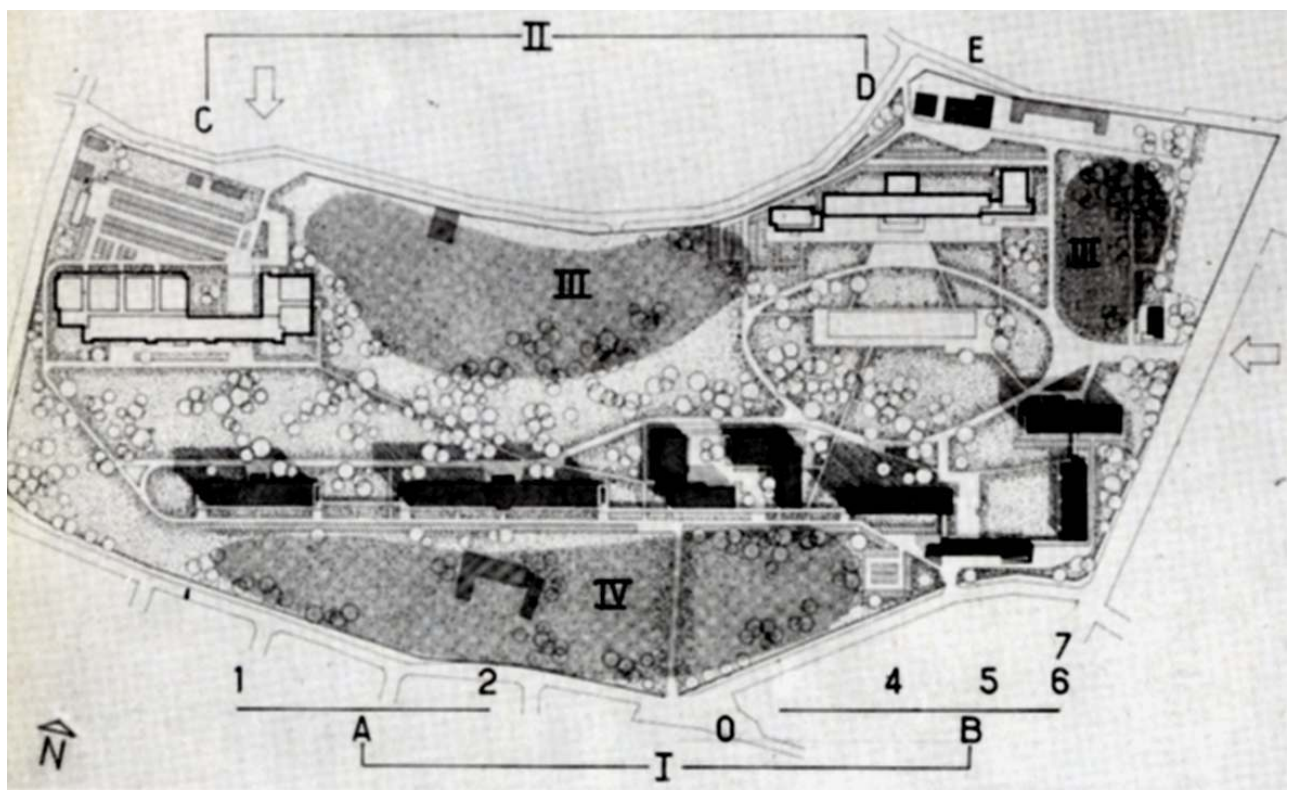

Plan de la faculté des sciences à Talence par René Coulon.

(C) CNAM/SIAF/CAPA/Archives d'architecture du XXe siècle, fonds DAU 133 IFA 70/1.

$9 \quad$ Le principe de séparation des disciplines scientifiques dans des édifices distincts figurait déjà dans le programme de reconstruction des années trente. Expert, dans son projet pour le cours de la Marne assignait à chaque discipline une des dents du grand plan en peigne qu'il proposait. L'affectation d'un ou plusieurs bâtiments à une discipline particulière est une caractéristique des projets de reconstruction des facultés des sciences françaises voire étrangères. Le principe se retrouve dans le projet de Louis Arretche pour la faculté de Rennes ${ }^{7}$, et dans une moindre mesure dans celui d'Henry Bernard pour celle de Caen. "Cette composition favorisait la volonté de discrimination [sic] entre les disciplines scientifiques et permettait de donner aux divers types de bâtiments recherche, travaux pratiques et enseignement théorique - une structure et des dispositions propres à leurs fonctions ${ }^{8}$. » Ce choix a permis de préserver pour l'agrément des usagers le secteur central de l'ancien parc ainsi que sa pièce d'eau (fig. $n^{\circ} 5$ ). 
Figure 5

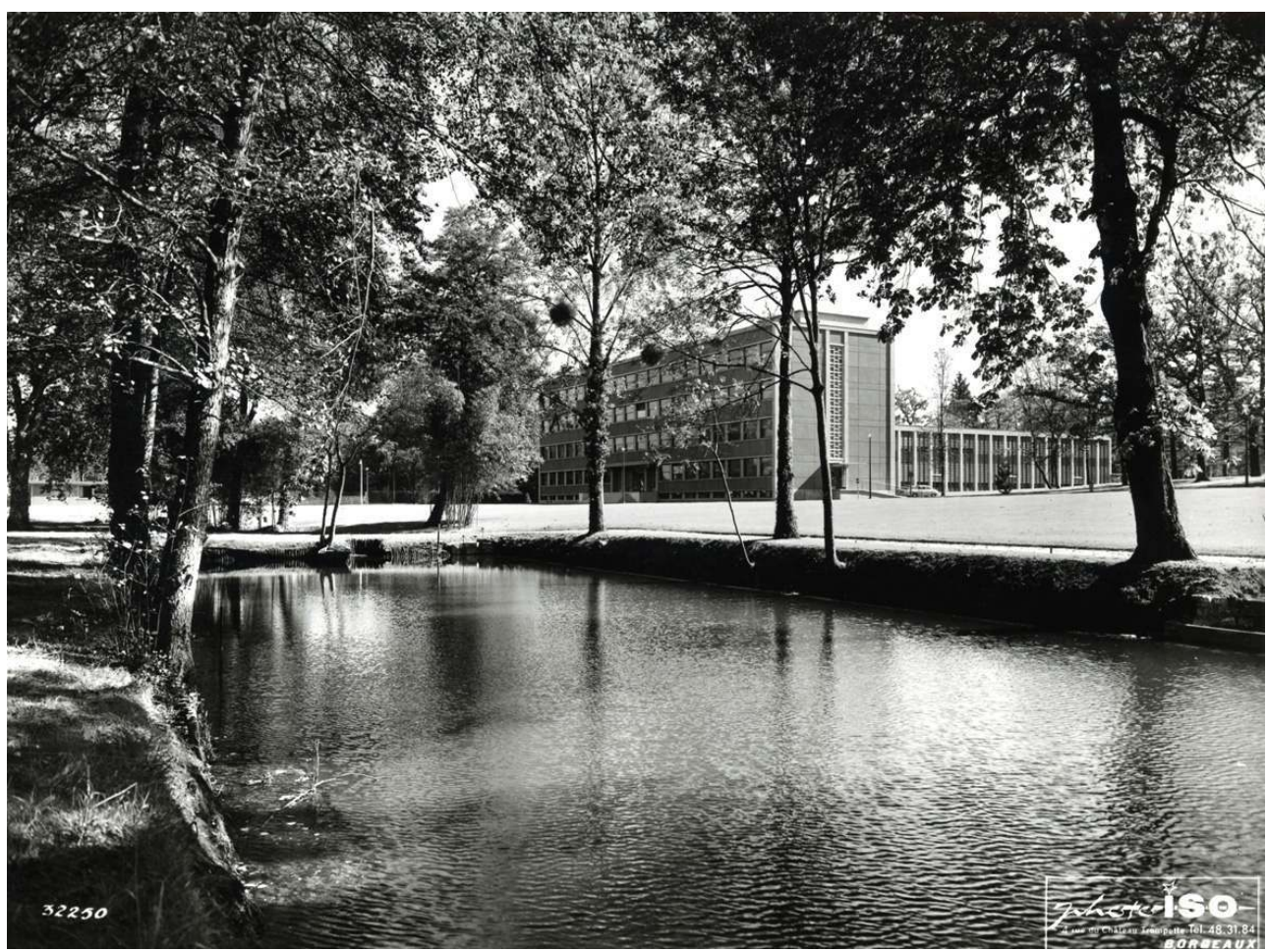

Bâtiment des travaux pratiques de physique. Photo industrielle du Sud-ouest.

(c) CNAM/SIAF/CAPA/Archives d'architecture du XXe siècle, fonds DAU 133 IFA 70/1.

10 Le projet d'ensemble de la faculté est soumis en 1953 à l'examen du Comité des architectes conseils de la ville9. L'architecte Jacques Carlu est chargé de rapporter le projet dans la séance du 5 mars 1953 de ce comité10. Dans son rapport, Carlu relate la présentation qu'en font Coulon et le Doyen Brus. Ceux-ci prétendent édifier « la faculté de l'An 2000, et (...) prévoir les extensions nécessaires ». Ils affirment qu'à l'achèvement de la faculté, le nombre d'étudiants, qui était de 617 en 1939, sera de 2000 en 1958. Le Doyen insiste sur l'urgence et la nécessité d'entreprendre rapidement des travaux ambitieux car «les facultés de Caen et de Dijon qui comptent moins d'étudiants sont en cours de reconstruction ${ }^{11} »$. On voit poindre le début de la mise en concurrence des grandes villes universitaires et régionales françaises qui sera une constante pendant les Trente glorieuses et encore aujourd'hui depuis les plans Université 2000.

11 L'édification s'effectue par tranches successives, ce qui permet, au fur et à mesure des livraisons de bâtiments, de déménager les différents départements des vieux locaux du centre ville. La première tranche comprend les départements de physique et de chimie ; la seconde, l'administration, propédeutique et les mathématiques; la troisième, l'école nationale supérieure de chimie; la quatrième, l'école supérieure d'électronique et de radioélectricité ; puis, nettement séparés de cette première phase de construction par la bibliothèque ${ }^{12}$, les instituts de zoologie, géologie et botanique (fig. $\mathbf{n}$ 6). 


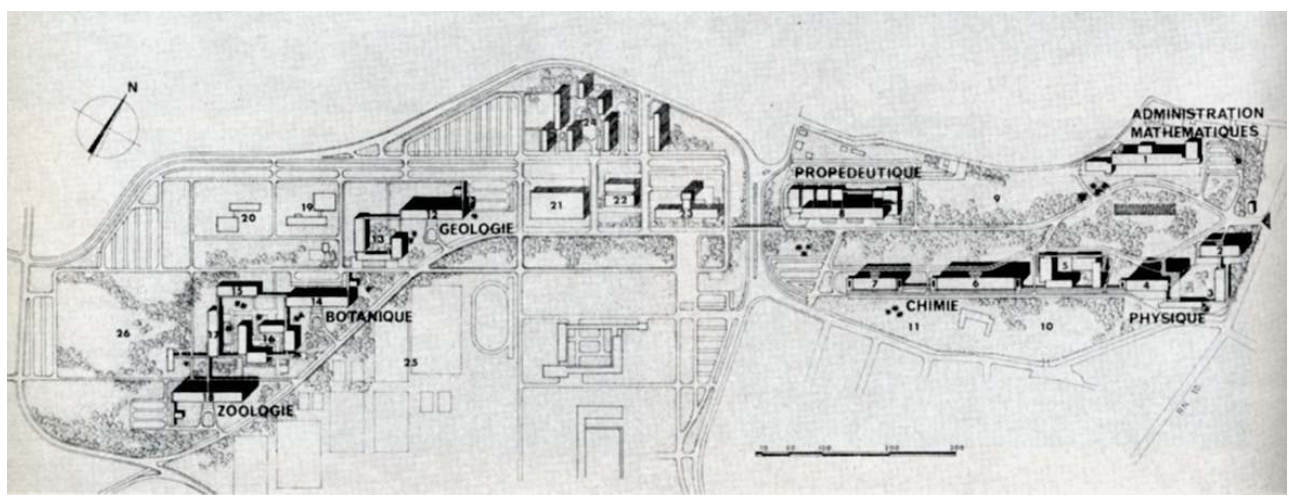

Plan des différentes tranches de la faculté des sciences.

(c) CNAM/SIAF/CAPA/Archives d'architecture du XXe siècle, fonds DAU 133 IFA 70/1.

\section{Le jeu avec les normes}

Carlu relaye, toujours dans son rapport de 1953, la proposition de Coulon d'adopter, en lieu et place du module de 1,75 m préconisé par le ministère de l'Éducation nationale pour les bâtiments d'enseignement, un module de $3 \mathrm{~m}$. L'application d'un module permettant l'élaboration de plans types de salles de classe pour l'enseignement du premier degré peut paraitre de prime abord un peu arbitraire pour des locaux d'enseignement supérieur. Une des préoccupations de la Direction des constructions scolaires, universitaires et sportives était de "dégager mieux chaque jour les stipulations fonctionnelles, dimensionnelles et techniques, et les normes de coût des constructions et de certains matériels » afin de pouvoir « obtenir malgré les contraintes du moment, des expressions architecturales satisfaisantes, et par là, éducatrices ${ }^{13}$ ». Pour les disciplines scientifiques, aux salles de cours se substituent des laboratoires aux besoins spécifiques et des amphithéâtres ne répondant pas eux-mêmes à des plans prédéfinis. D'où, on le comprend, la souplesse toute relative laissée aux architectes.

La proposition de Coulon de recourir à un autre module, d'ailleurs adoptée par le conseil municipal de Bordeaux, est justifiée d'un point de vue esthétique par l'architecte luimême. Pour lui, «l'ordonnancement des façades avec le module de trois mètres est très supérieur aux façades conçues avec le module d'1,75 $\mathrm{m}$ ou ses multiples et sous-multiples ${ }^{14}$. » Le projet ayant alors besoin d'être soutenu par les autorités en vue de son examen par le Conseil général des Bâtiments de France, le secrétaire général de la mairie s'adresse par note écrite au maire pour l'avertir «qu'il y aurait lieu d'intervenir auprès de $\mathrm{M}$. André Marie, ministre de l'Éducation nationale et du Directeur des constructions scolaires 15 ».

Chaban-Delmas a très certainement plaidé la cause de l'université bordelaise auprès de son collègue ministre puisque celui-ci lui répond par écrit le 29 avril 1953: «En te renvoyant les lettres de M. le Recteur Delage et de M. Chevalier, inspecteur général, chef du service technique des constructions, que tu m'avais communiquées, je crois pouvoir te dire que je considère l'affaire soulevée par l'adoption d'un module de $3 \mathrm{~m}$ pour la construction de la nouvelle faculté des Sciences de Bordeaux comme terminée ${ }^{16}$.» 
L'intervention politique a ainsi probablement permis l'adoption de solutions constructives qui feront la preuve de leur bien-fondé tant fonctionnel qu'esthétique.

En l'absence de sources plus précises sur l'étude et la construction de la faculté des sciences de Bordeaux - notamment l'accès aux archives de l'architecte - on peut donc dater le projet de René Coulon de l'année $1953^{17}$. Les travaux de la première tranche sont mis en adjudication le 2 décembre 1955. C'est l'entreprise parisienne de maçonnerie Pérignon qui remporte le lot principal de gros œuvre. Les architectes d'opération sont R. et J. Touzin. Les premiers bâtiments sont livrés en 1961 (fig. $\mathbf{n}^{\circ} 7$ ).

Figure 7

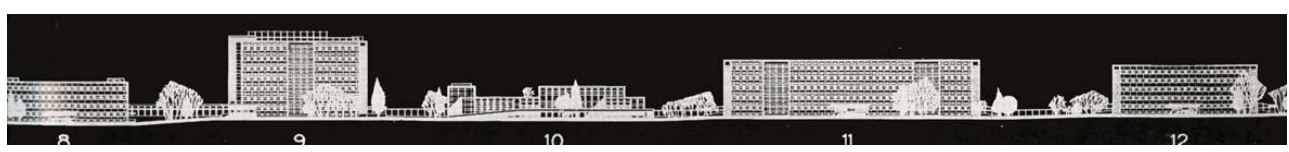

Élévation générale de la première tranche de la faculté des Sciences par René Coulon.

(c) CNAM/SIAF/CAPA/Archives d'architecture du XXe siècle, fonds DAU 133 IFA 70/1.

\section{L'architecture rationaliste de Coulon}

Les huit bâtiments qui composent la première tranche sont implantés le long d'une ligne orientée est-ouest qui rappelle la voie de même orientation qui traverse le plan d'ensemble de la ville nouvelle de Mourenx près de Pau, dessiné par Coulon ${ }^{18}$ (fig. $\mathbf{n}^{\circ} \mathbf{8}$ ). Cette ligne matérialisée par une circulation remplit le rôle d'axe structurant et détermine l'orientation des bâtiments dont les plus grandes façades s'ouvrent au nord et au sud. À l'ouest, se situe le département chimie et à l'est, le département physique dont les bâtiments disposés en équerre marquent la limite du domaine vers Bordeaux et en forment l'accès principal, marqué plus tard par la grille monumentale dessinée par Raymond Subes. Entre les deux groupes, les amphithéâtres communs sont répartis dans deux équerres inversées se joignant comme deux mains (fig. $\left.\mathbf{n}^{\circ} \mathbf{9}\right)$. Des galeries couvertes constituées par des dalles portées par de fins poteaux de béton armé, protègent les circulations extérieures et les liaisons entre les bâtiments (fig. n 10). 
Figure 8

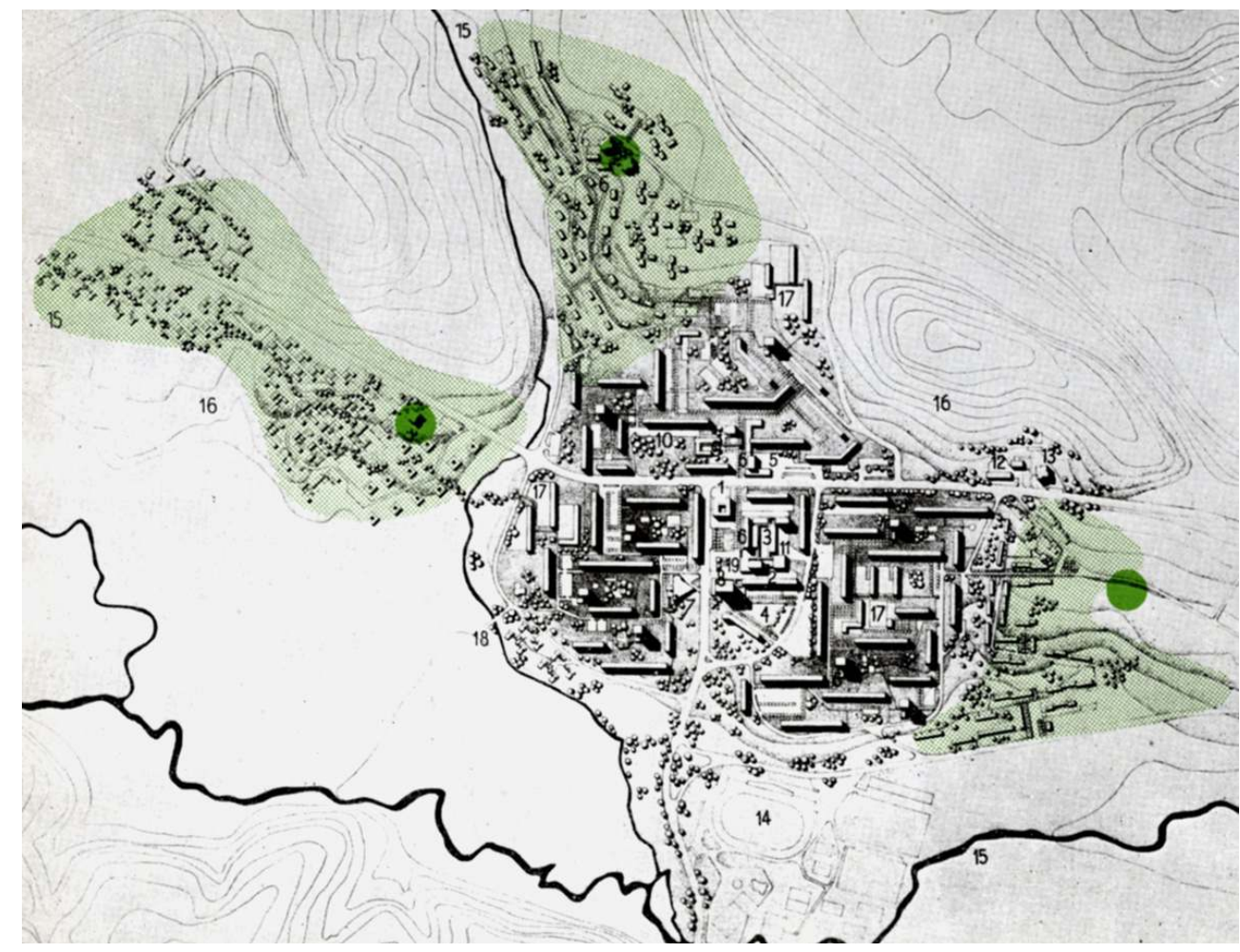

Plan de masse de la ville nouvelle de Mourenx dans les Pyrénées-Atlantiques par René Coulon.

(C) CNAM/SIAF/CAPA/Archives d'architecture du XXe siècle, fonds DAU 133 IFA 70/1. 


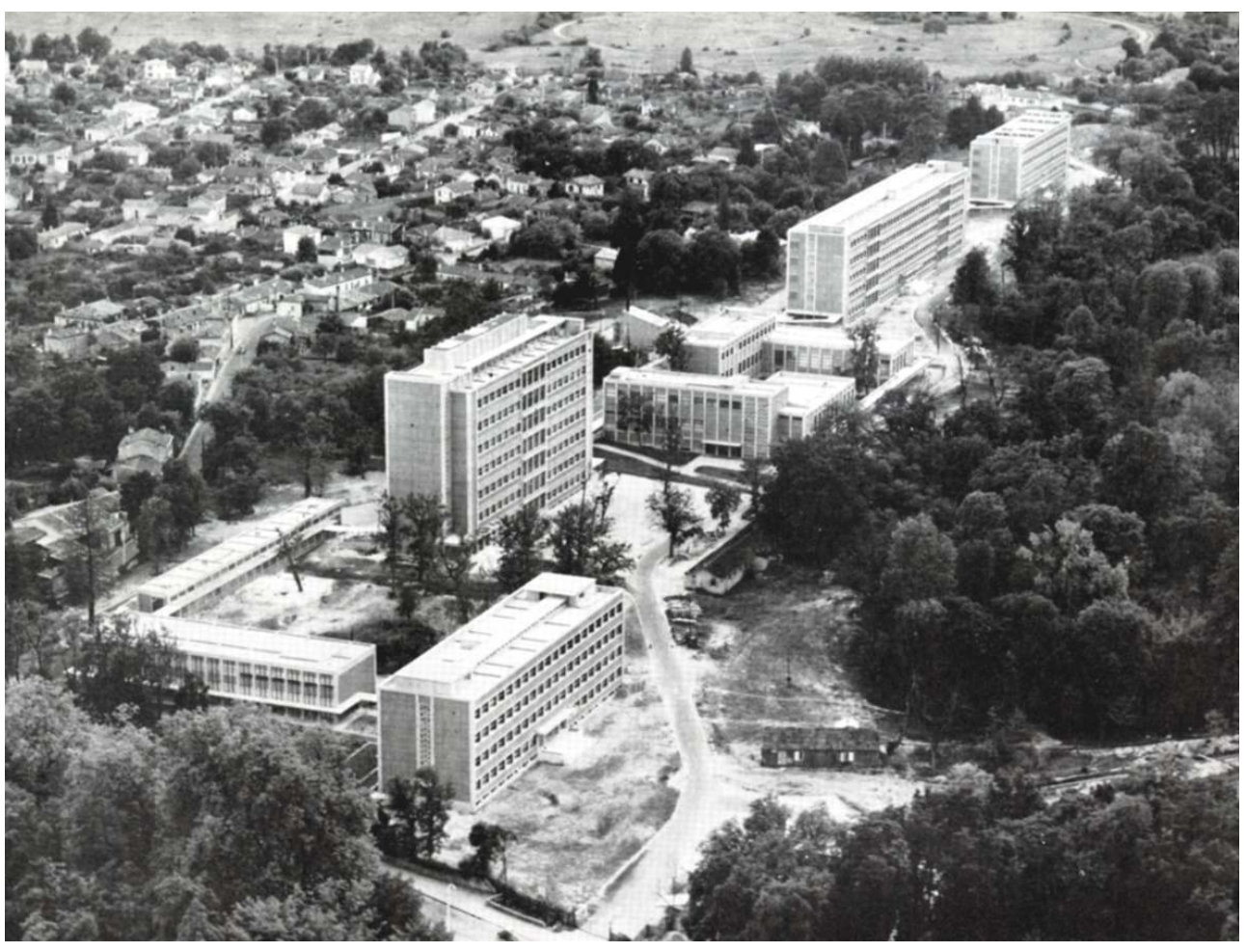

Vue aérienne de la première tranche de la faculté des sciences, cliché anonyme.

(c) CNAM/SIAF/CAPA/Archives d'architecture du XXe siècle, fonds DAU 133 IFA 70/1.

\section{Figure 10}

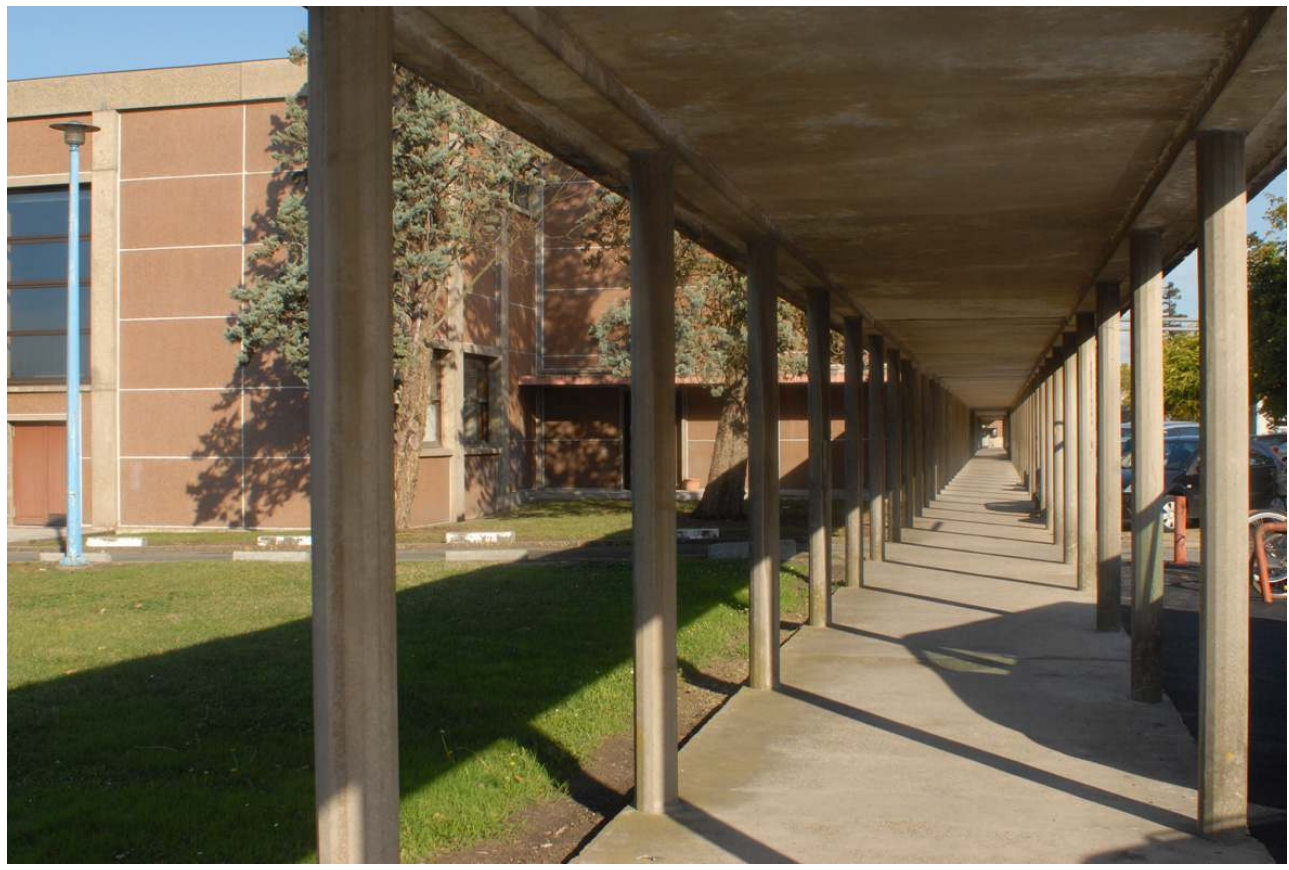

Galerie de liaison couverte.

Phot. Michel Dubau. (c) Service régional du patrimoine et de l'inventaire d'Aquitaine, 2008. 
17 Tous les bâtiments, à la silhouette parallélépipédique, sont fortement rythmés par les trumeaux séparant les ouvertures (fig. $\mathbf{n}^{\mathbf{0} 11}$ ). Les lignes hautes et basses des allèges sont soulignées de bandeaux continus. Pareillement, les arêtes extérieures des édifices sont marquées par de larges bandeaux plats sur toute la hauteur et toute la longueur, délimitant fortement les volumes construits à la manière de chaînages. Les circulations verticales sont identifiables sur les élévations par la présence de grands claustras de béton (fig. $\mathbf{n}^{\circ} \mathbf{1 2}$ ). L'horizontalité des deux barres de quatre étages renfermant les laboratoires de chimie est accentuée par les fenêtres en longueur. Aux angles des façades sud, des brise-soleil faits de lames de béton horizontales se retournent sur les pignons pour former un motif d'angle (fig. $\mathbf{n}^{\circ} \mathbf{1 3}$ ).

Figure 11

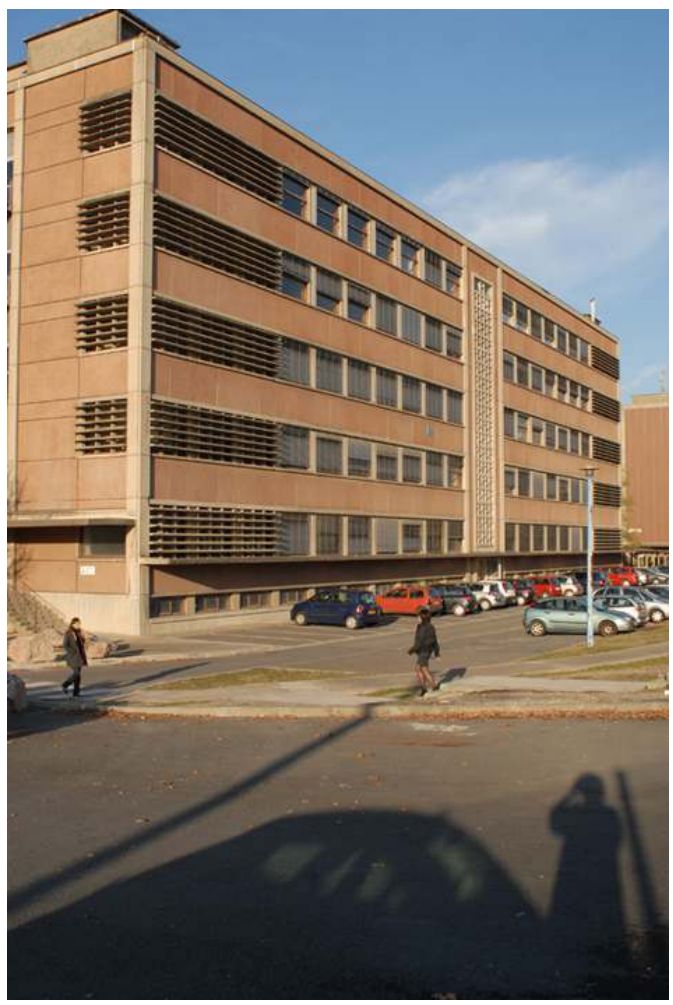

Façade sud d'un bâtiment de laboratoires et travaux pratiques de chimie.

Phot. Michel Dubau. (c) Service régional du patrimoine et de l'inventaire d'Aquitaine, 2008. 
Figure 12

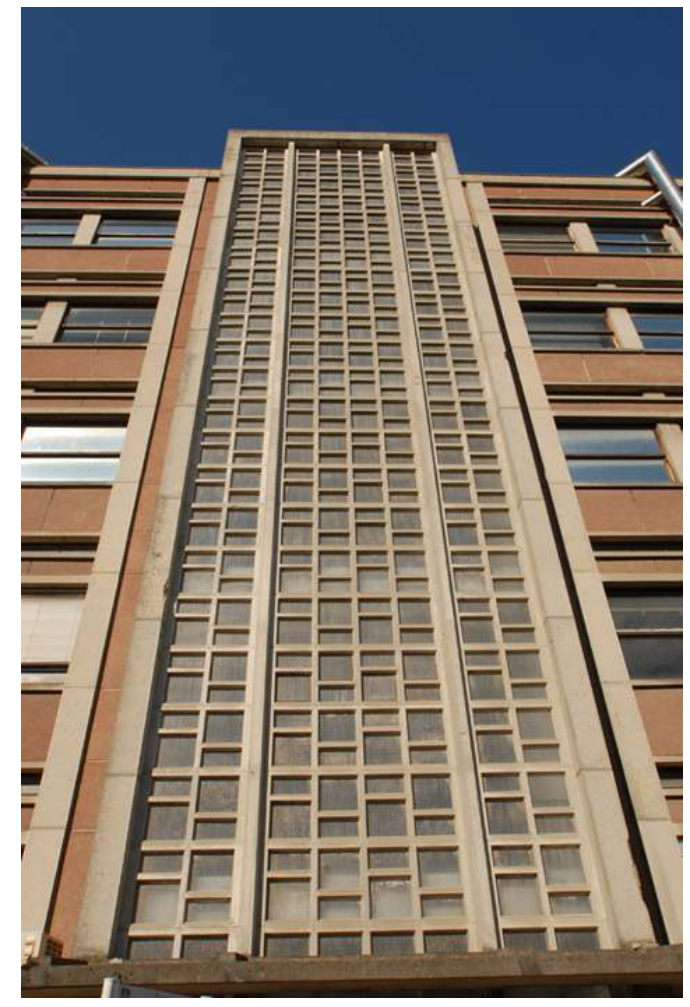

Claustra sur la façade d'un bâtiment de laboratoires et travaux pratiques de chimie. Phot. Michel Dubau (c) Région Aquitaine, Inventaire général, 2008. 
Figure 13

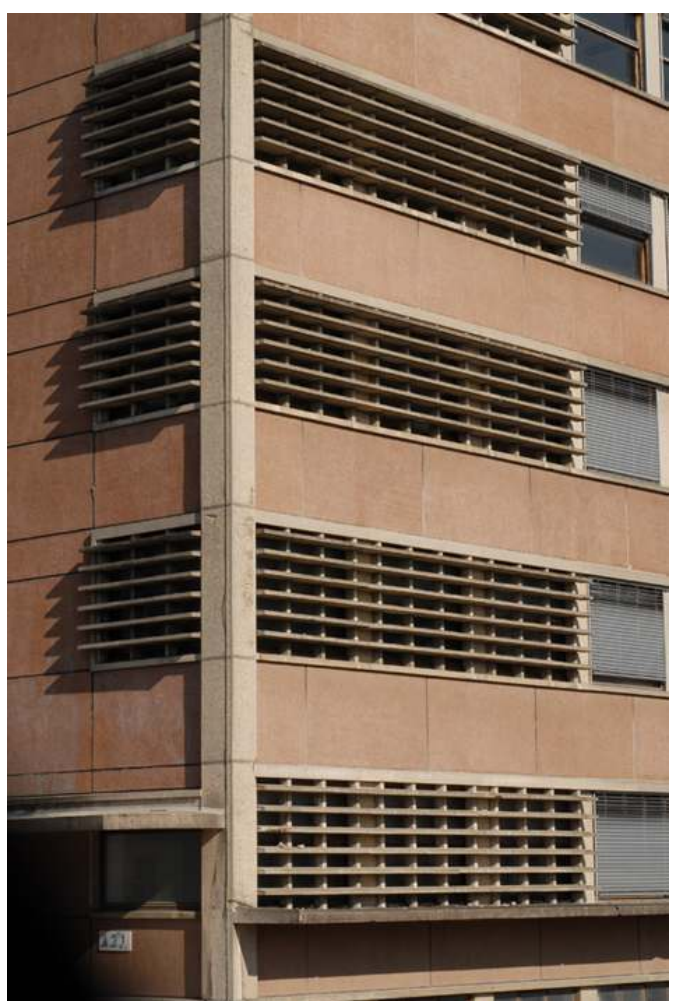

Brise soleil à l'angle d'un bâtiment de laboratoires et travaux pratiques de chimie.

Phot. Michel Dubau. (c) Service régional du patrimoine et de l'inventaire d'Aquitaine, 2008.

18 Philippe Dehan estime que « comme Jean Ginsberg, René-André Coulon fait partie d'une génération pour laquelle l'architecture moderne n'est plus un combat ou une doctrine, mais un ensemble de principes à exploiter sans renier les traditions, qu'il s'agisse des règles de l'art ou des principes de composition ${ }^{19}$. " La façade est ainsi fortement et symétriquement composée par un motif central et deux motifs latéraux traités à la manière d'avant-corps (fig. $\mathbf{n}^{\circ} \mathbf{1 4}$ ). Un réseau de joints creux dessine une grille sur l'ensemble des élévations. La figure de la grille est très présente dans l'œuvre de Coulon, mais de manière discrète et fine. 
Figure 14

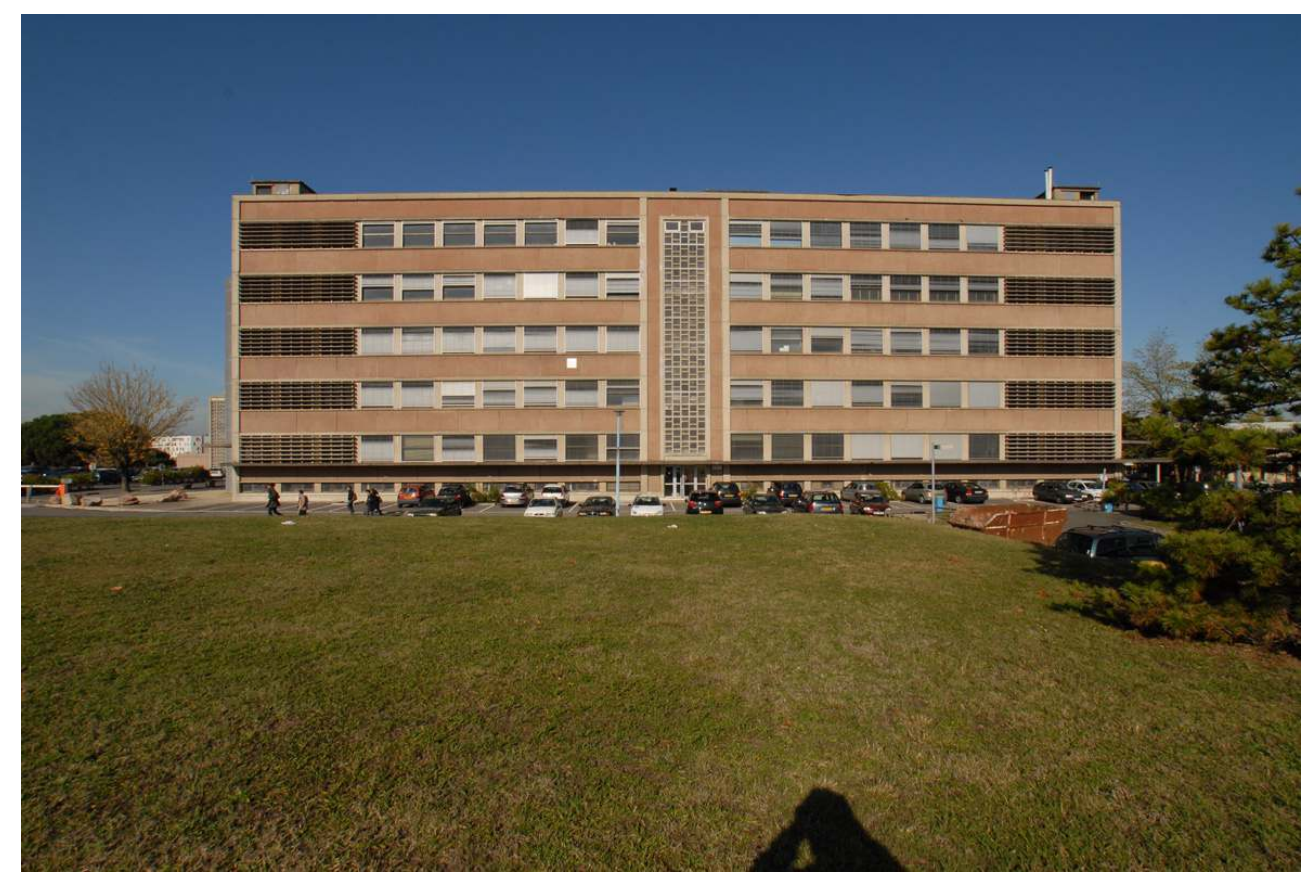

Façade sud d'un bâtiment de laboratoires et travaux pratiques de chimie.

Phot. Michel Dubau. (c) Service régional du patrimoine et de l'inventaire d'Aquitaine, 2008.

\section{Une modernité monumentale}

19 Ce motif de la grille provient presque toujours chez Coulon de la structure porteuse et des trames constructives adoptées. Elle rythme ainsi fortement, par exemple, les façades de la crèche et du centre social Renault à Boulogne-Billancourt où les poteaux de béton armé et les nez de dalle forment des cadres dans lesquels viennent s'inscrire les fines menuiseries métalliques des baies vitrées divisant la travée en quatre.

Dans les bâtiments de la faculté de Bordeaux, la structure porteuse rythme les façades des amphithéâtres. Les vides entre poteaux sont comblés par des panneaux préfabriqués de béton (fig. $\left.\mathbf{n}^{\circ} \mathbf{1 5}\right)$. Les laboratoires sont recouverts des mêmes panneaux revêtant la structure mais celle-ci est suggérée en façade par des joints creux qui en reprennent exactement la trame. Par cette solution de revêtement, les constructions se rapprochent des deux premiers bâtiments de la faculté des sciences de Jussieu à Paris que Coulon édifie à la même époque avec Urbain Cassan, Louis Madeline, et Roger Séassal ${ }^{20}$. 
Figure 15

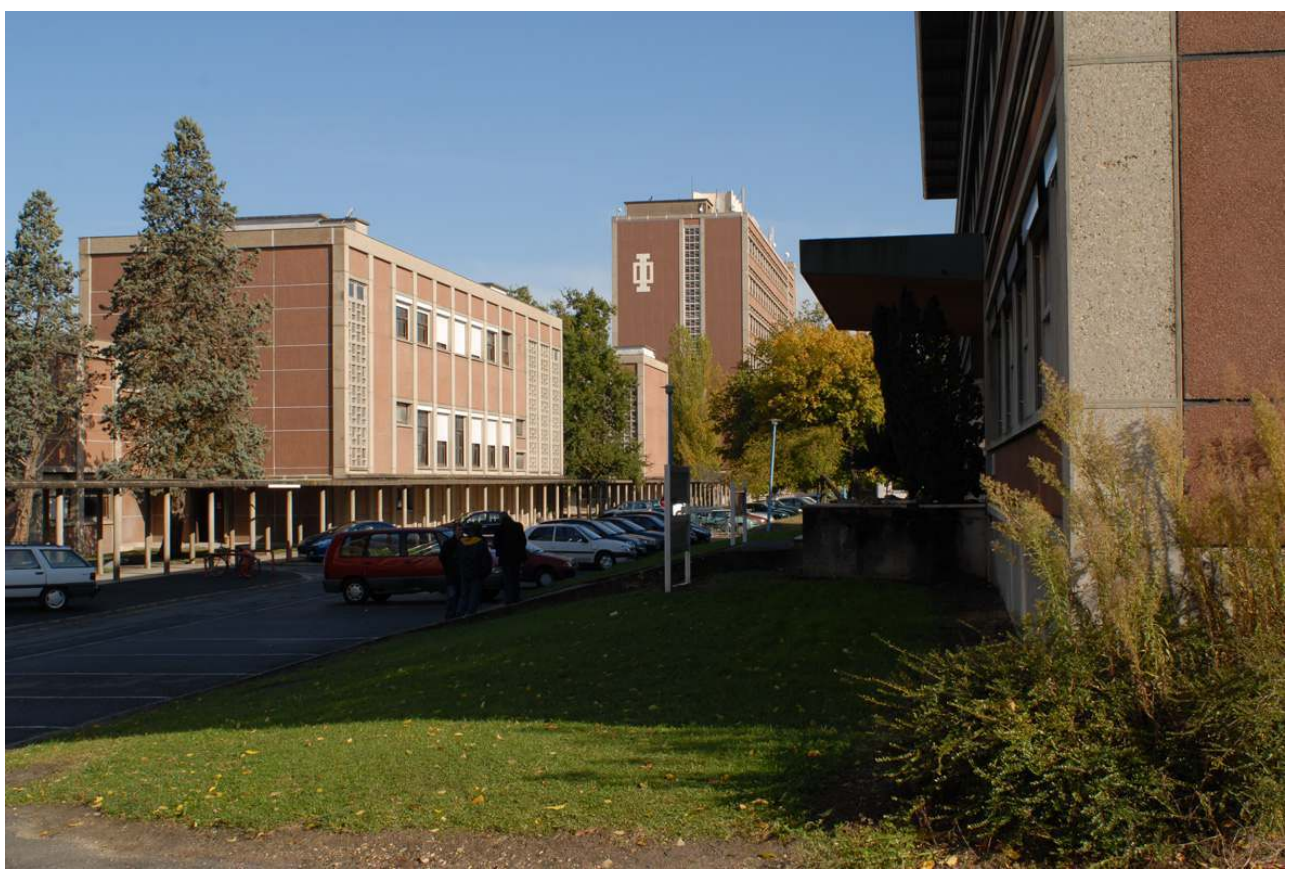

Bâtiments des amphithéâtres.

Phot. Michel Dubau. (c) Service régional du patrimoine et de l'inventaire d'Aquitaine, 2008.

21 À Bordeaux, la trame plus serrée du bâtiment de l'administration de la faculté rappelle davantage celle des immeubles de bureaux construits par Coulon, notamment celui du siège administratif d'EDF à Paris, et en particulier la façade sur la rue Murat (fig. $\mathbf{n}^{\circ} \mathbf{1 6}$ ). En dehors des trumeaux filant sur toute la hauteur, l'élévation sud est largement vitrée. Le jeu d'opposition entre le rythme vertical des travées et celui horizontal des fenêtres et des allèges engendre une façade équilibrée dans les deux directions sans être rébarbative (fig. $\mathbf{n}^{\circ} \mathbf{1 7}$ ). 
Figure 16

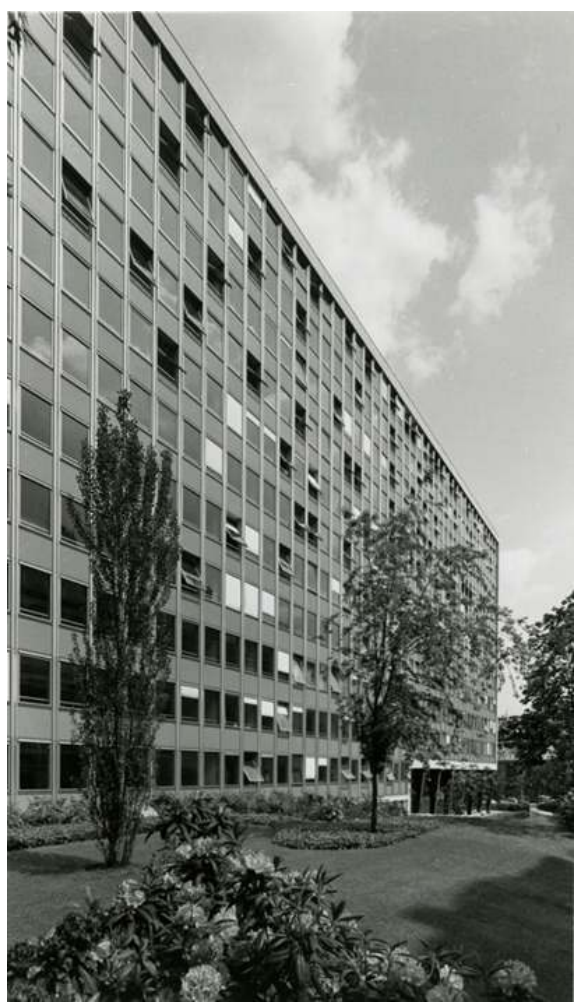

Siège social d'EDF à Paris par René Coulon, façade sur la rue Murat.

Henrot. () CNAM/SIAF/CAPA/Archives d'architecture du XXe siècle, fonds DAU 133 IFA 70/1, 1965.

\section{Figure 17}

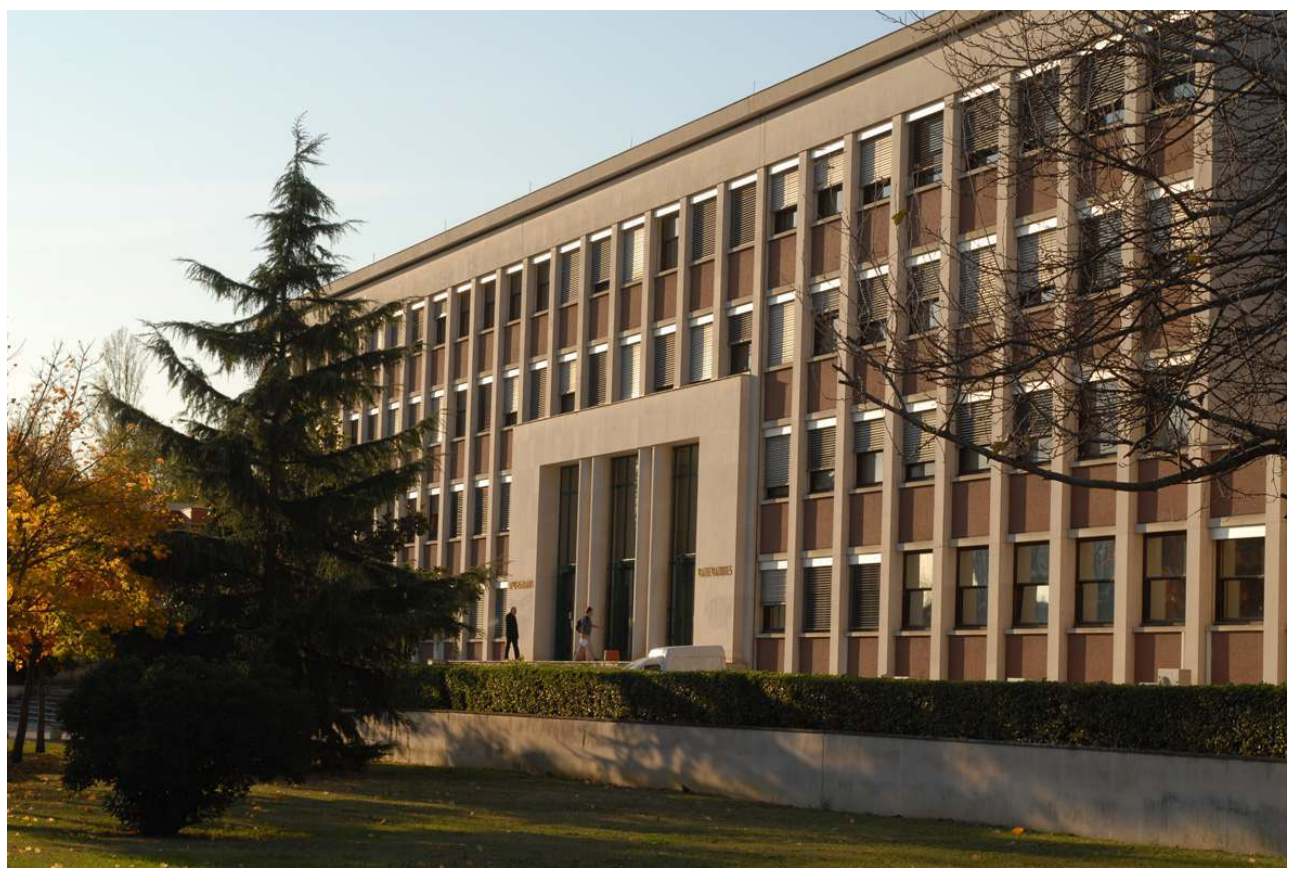

Façade sud du bâtiment de l'administration de la faculté.

Phot. Michel Dubau. @ Service régional du patrimoine et de l'inventaire d'Aquitaine, 2008. 
Tous ces jeux de trame et de grille confèrent aux bâtiments bordelais de Coulon et à l'ensemble qu'ils forment une puissance et une grandeur un peu froides mais tempérées par le traitement coloré des matériaux et revêtements. Les bandeaux verticaux et horizontaux sont en béton bouchardé. Les panneaux de remplissage sont en béton lavé à gravillons concassés rouges (fig. $\mathbf{n}^{\circ} \mathbf{1 8}$ ).

Figure 18

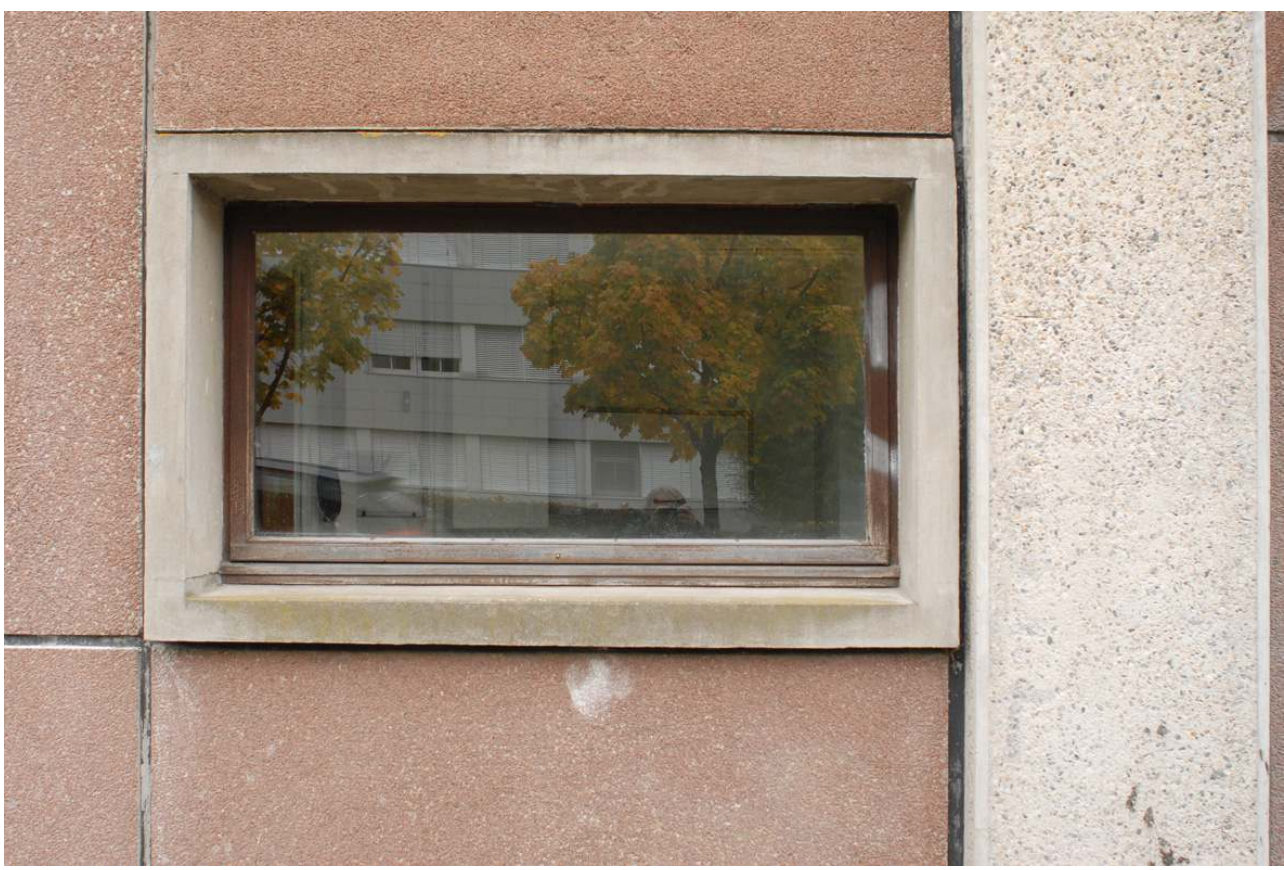

Détail de façade.

Phot. Michel Dubau. (@) Service régional du patrimoine et de l'inventaire d'Aquitaine, 2008.

Par des principes de composition simples, l'architecte réussit à conférer à la faculté un caractère et une expression qui siéent bien à des édifices publics et universitaires. "L'architecture, très orthogonale, plate et assez uniforme, reste à l'échelle humaine et sa simplicité n'exclut pas les proportions et une certaine diversité dans la composition des façades. » Cette description, due à René Jullian, de la ville nouvelle de Mourenx peut tout à fait s'appliquer à la faculté des sciences ${ }^{21}$. Le bâtiment de l'administration trahit une référence certaine aux édifices universitaires bordelais de la fin du XIX siècle. La façade, bien qu'uniforme, est couronnée par un épais bandeau d'acrotère avec ressaut lisse et sans modénature qui évoque la figure de la corniche. L'entrée traitée en forme de porche en léger avant-corps, est ponctuée de deux paires de poteaux cylindriques sans base ni chapiteaux, mais rappelant des colonnes (fig. $\mathbf{n}^{\circ}{ }^{\circ}$ ). La rupture d'échelle voulue entre ce motif d'entrée et le reste de la façade introduit une monumentalisation de l'entrée. 
Figure 19

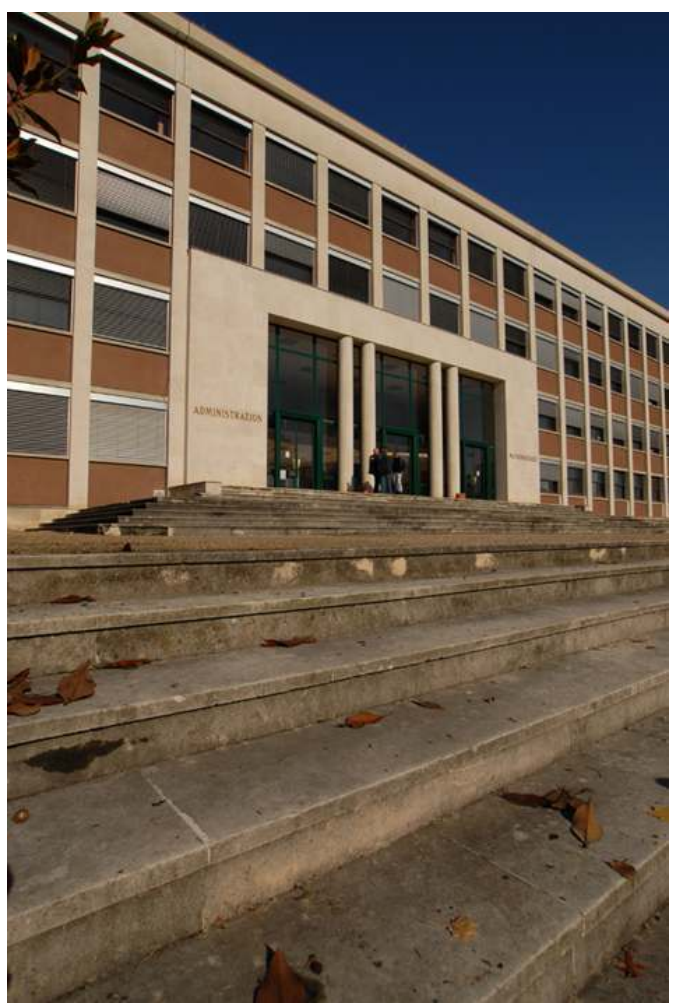

Entrée du bâtiment de l'administration.

Phot. Michel Dubau. (c) Service régional du patrimoine et de l'inventaire d'Aquitaine, 2008.

\section{Architecture et industrie}

Si Coulon porte un soin attentif à l'aspect extérieur des constructions, les espaces intérieurs ne sont pas pour autant négligés, malgré le contexte économique difficile du tout début des années cinquante. Là aussi, des solutions simples, efficaces et belles sont retenues. Les cages d'escalier offrent leurs larges et amples volées enroulées autour d'un vide central le long duquel court le ruban du haut limon surmonté d'un garde-corps en barreaux fins et main courante gainée (fig. $\mathbf{n}^{\circ} \mathbf{2 0}$ ). Les cages sont baignées de lumière par des claustras de béton montant de fond et garnis de verre armé. Il faut rappeler ici que Coulon avait été l'auteur avec Jacques Adnet à l'Exposition internationale de $1937 \mathrm{du}$ surprenant pavillon des verreries de Saint-Gobain, construit entièrement en verre. L'enveloppe et les planchers du pavillon étaient réalisés en panneaux de briques de verre suspendus à l'ossature de poteaux et poutres en béton. Pour Philippe Dehan, «ce bâtiment démontre de manière optimiste la fécondité de la collaboration entre architecture et industrie. Un crédo auquel René-André Coulon restera fidèle toute sa carrière $^{22}$.» Ce dont témoigne la faculté des sciences tant par son architecture que dans les détails de sa mise en œuvre. 
Figure 20

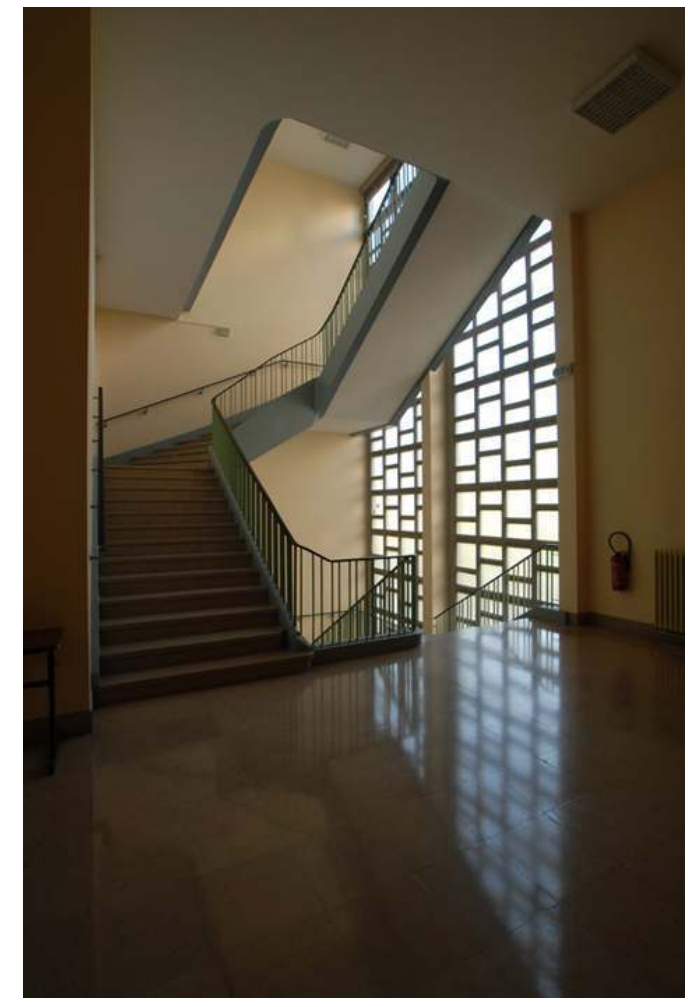

Cage d'escalier.

Phot. Michel Dubau. (c) Service régional du patrimoine et de l'inventaire d'Aquitaine, 2008.

Le travail sur les menuiseries intérieures est également remarquable. Les doubles portes battantes de communication sont en panneaux sans autre détail que les veines du placage de bois. Leurs grandes poignées en métal gainé suffisent à l'animation et au graphisme (fig. $\mathbf{n}^{\circ} \mathbf{2 1}$ ). À l'intérieur des amphithéâtres, au-dessus des portes d'accès, un panneau de menuiserie reprend dans son dessin de baguettes le dessin du réseau des claustras des cages d'escaliers, mais à l'horizontale (fig. $\mathbf{n}^{\circ} \mathbf{2 2}$ ). 
Figure 21

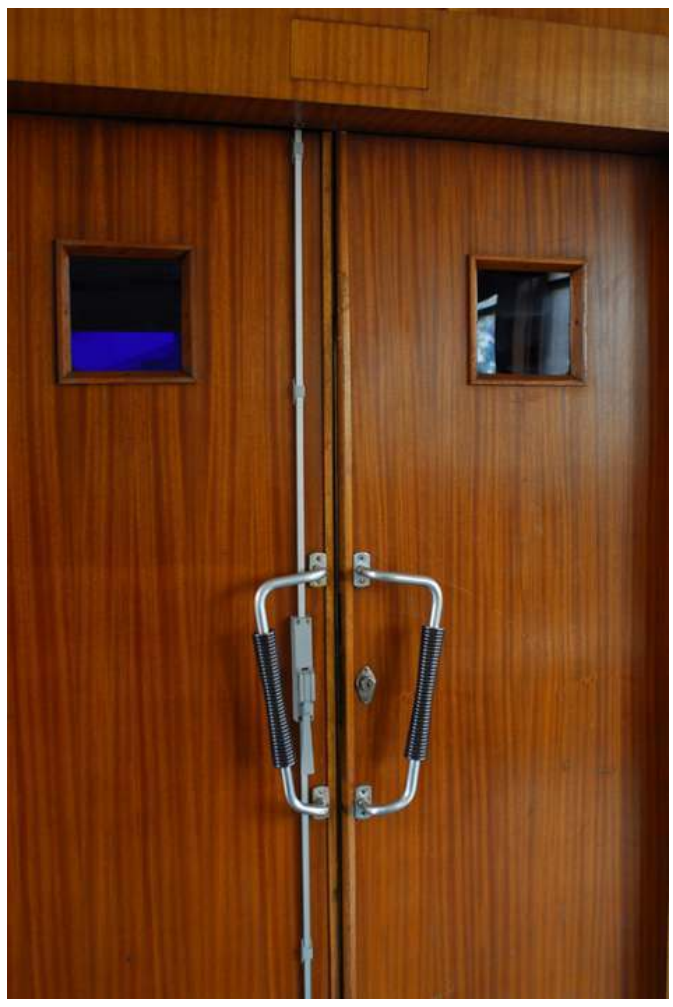

Porte d'accès à un amphithéâtre.

Phot. Michel Dubau. (c) Service régional du patrimoine et de l'inventaire d'Aquitaine, 2008.

\section{Figure 22}

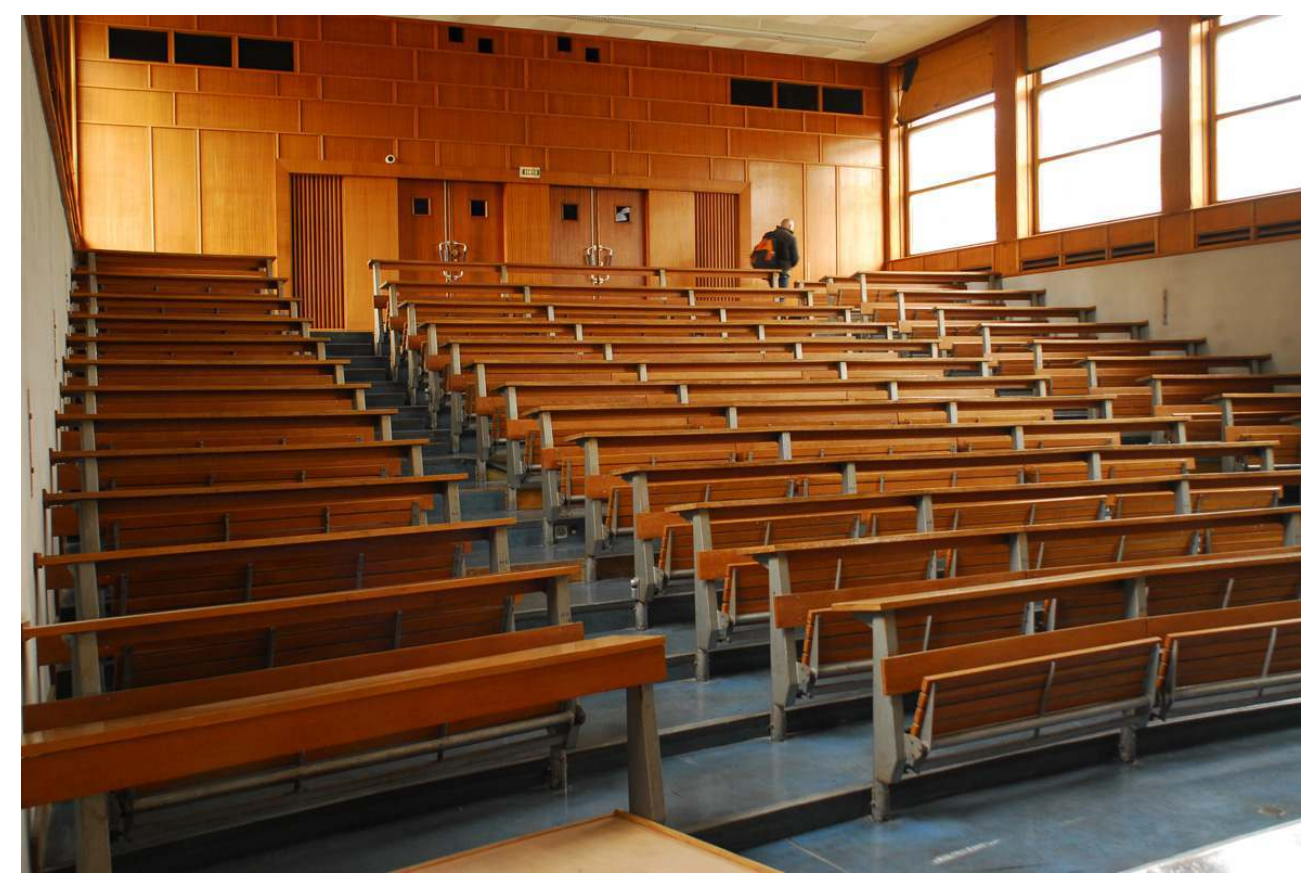

Intérieur d'un amphithéâtre.

Phot. Michel Dubau. (c) Service régional du patrimoine et de l'inventaire d'Aquitaine, 2008. 
Depuis 1937, Coulon s'est fait ainsi le chantre d'une architecture utilisant les techniques modernes et les produits de l'industrie en n'oubliant pas pour autant les leçons de composition dont il fut nourri à l'École des beaux-arts de Paris dans l'atelier Defrasse et Madeline. De Coulon, Ionel Schein a fait au début des années soixante le portrait suivant: "Efficace, précis, imperturbable, ayant le sentiment que l'architecture se pré-détermine autant qu'elle se construit, calculant chaque risque, il oriente l'architecture des organismes les plus importants et réalise des ensembles où la primauté technologique est presque toujours exprimée ${ }^{23}$.»

\section{Coulon architecte spécialiste des « campus »}

La faculté des sciences de Bordeaux est très représentative du travail de Coulon en matière de «campus » de recherche ou d'enseignement supérieur. En 1958, il édifie à Bagneux pour la Compagnie française Thomson-Houston des laboratoires organisés en peigne de part et d'autre d'un bâtiment qui fait office d'épine dorsale. Les bâtiments sont reliés entre eux par de légères galeries couvertes et vitrées. La construction fait, cette fois, plus largement appel au métal qu'au béton et l'utilisation d'éléments préfabriqués est poussée plus avant (fig. $\mathbf{n}^{\circ} \mathbf{2 3}$ ).

Figure 23

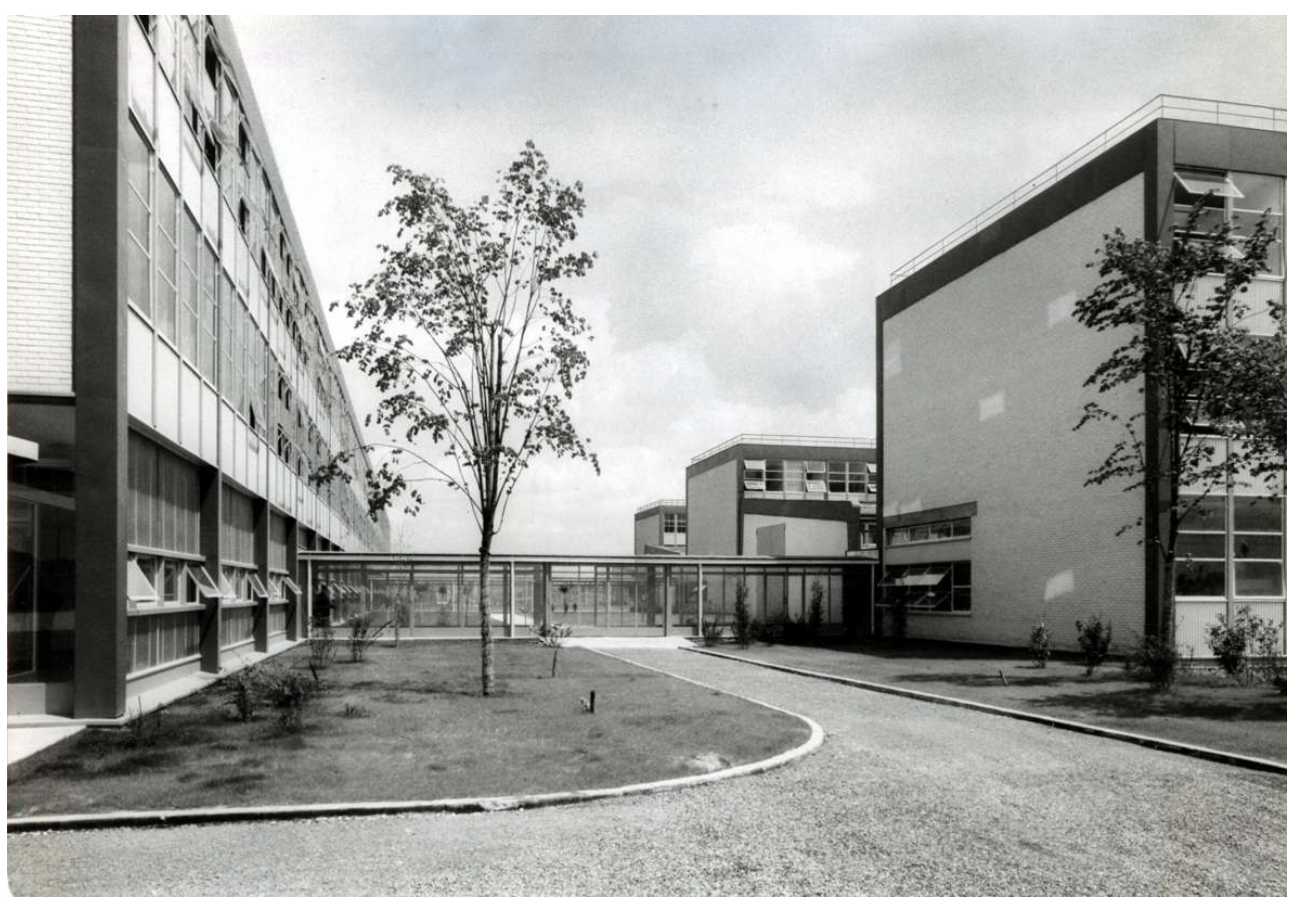

Laboratoires de la Thomson-Houston à Bagneux par René Coulon.

Henrot ( ) CNAM/SIAF/CAPA/Archives d'architecture du XXe siècle, fonds DAU 133 IFA 70/1, 1958

Pour le transfert de l'École des Hautes études commerciales (HEC) à Jouy-en-Josas en 1961, Coulon dissémine, dans un grand parc vallonné et boisé de 110 ha en bordure de la Bièvre, un ensemble dont «la construction est personnalisée suivant la fonction des bâtiments ", reprenant ainsi l'organisation déjà retenue pour la faculté des sciences de Bordeaux (fig. $\mathbf{n}^{\circ} \mathbf{2 4}$ ). «Le zonage des constructions distingue : l'administration et la réception, les études, l'habitat, les services annexes, les sports ${ }^{24}$.» 


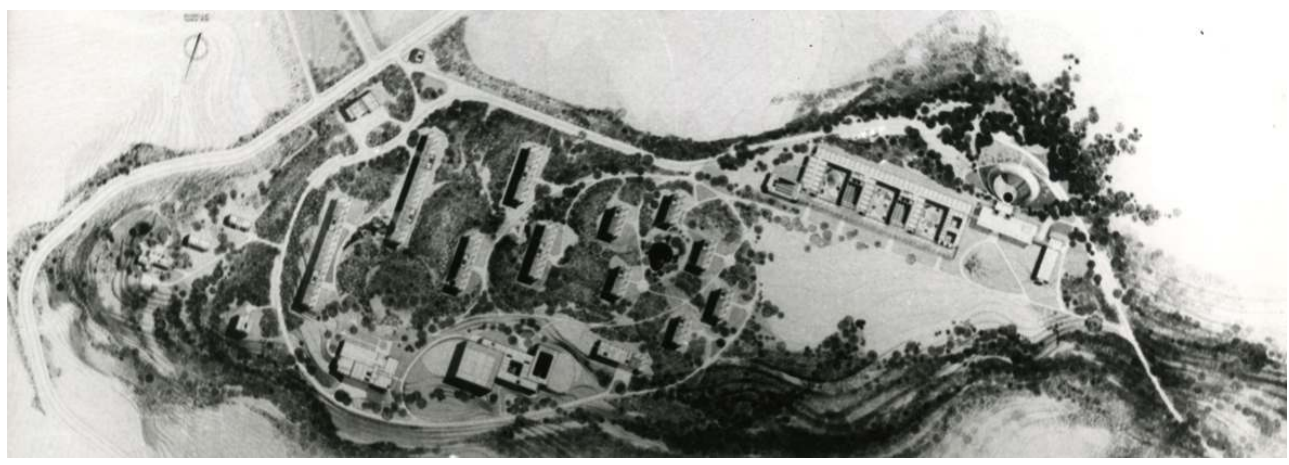

Plan de masse de l'École des Hautes études commerciales à Jouy-en-Josas par René Coulon. (c) CNAM/SIAF/CAPA/Archives d'architecture du XXe siècle, fonds DAU 133 IFA 70/1.

La maitrise de la grande échelle et l'intérêt pour l'application de l'industrialisation dans l'architecture font de Coulon l'auteur de nombreux immeubles de bureaux publics ou privés. Ces deux aspects - ajoutés à son statut d'architecte en chef des Bâtiments civils et palais nationaux le désignent, avec son confrère Édouard Albert, comme les deux chefs de l'équipe de maîtres d'œuvre chargés à partir de 1964 de la construction de la faculté des sciences de Jussieu. En plein cœur de Paris, le parti architectural radical impose la rigueur de son plan en gril et de ses façades en murs-rideaux. Pour autant, la monumentalité n'est pas abandonnée. Elle se manifeste notamment dans la figure de l'entrée marquée par l'interruption de la barre ; au travers de la faille se profile l'arrête verticale de la tour de l'administration.

L'architecture de René-André Coulon illustre bien le surgissement d'ensembles et de bâtiments qui, par leur modernité, vont frapper les esprits et symboliser la transformation du paysage architectural et l'équipement du territoire français. L'irruption de la faculté des sciences dans l'espace encore très rural de la commune de Talence a certainement marqué un tournant. Elle a frappé l'œil artiste du peintre bordelais Georges de Sonneville qui en immortalisa le chantier en $1958^{25}$.

\section{NOTES}

1. - RAMBERT, Charles. Constructions scolaires et universitaires. I. Paris: Vincent, Fréal et Cie éditeurs, collection L'architecture française de nos jours, 1955, p. 7.

2. - Sur les bâtiments universitaires bordelais de la fin du XIXe siècle voir : DUSSOL, Dominique et LAROCHE, Claude. "Les facultés de Bordeaux». Dans RIVÉ, Philippe (dir.). La Sorbonne et sa reconstruction. Paris : La Manufacture, 1987, p. 201-222. La faculté de médecine et de pharmacie fait l'objet d'un développement dans LAROCHE, Claude. "Pro Scientia Urbe et Patria: l'architecture de la faculté de médecine et de pharmacie de Bordeaux, 1876-1888 et 1902-1922». Revue archéologique de Bordeaux, tome LXXXIII, 1992, p. 137-173. Voir également dans ce numéro : «Claude Laroche, «Pro Scientia Urbe et Patria : l'architecture de la faculté de médecine et de 
pharmacie de Bordeaux, 1876-1888 et 1902-1922 », In Situ [En ligne], 17 | 2011, mis en ligne le 24 janvier 2012, consulté le 26 janvier 2012. URL : http://insitu.revues.org/1126; DOI : 10.4000/ insitu.1126».

3. - Le bâtiment de l'institut de zoologie existe toujours de nos jours. Malgré son caractère éclectique, il témoigne d'une conception novatrice sur le plan de l'usage rationnel des différents matériaux (brique, fer et verre). L'entrée, située dans le pan coupé à l'angle du cours de la Marne et de l'ancien cours Barbey, affirme son statut de monument public par ses pilastres, sa grande arcade et son haut pignon. Actuellement désaffecté, l'institut de zoologie est menacé de démolition. Les éléments sur le projet de Lacombe nous ont été aimablement communiqués par Sylvain Schoonbaert de la Mission recensement du paysage architectural et urbain à la Direction générale de l'aménagement de Bordeaux.

4. - SAVES, Véronique. Les deux domaines universitaires de Bordeaux. Maîtrise sous la direction de Robert Coustet, Université de Bordeaux III, 1992 (disponible aux archives municipales de Bordeaux, fonds Coustet, $16 \mathrm{~S} 70$ ).

5. - DELASNE, Sabine. «Illusions et désillusions des premiers campus en France ». Dans POIRRIER, Philippe (dir.). Paysage des campus. Urbanisme, architecture et patrimoine. Dijon: Presses universitaires de Dijon, 2009, p. 42-49.

6. - « Nouvelle faculté des sciences de Bordeaux ». L'Architecture française, n²31-232, novembredécembre 1961, p. 18-23. Le texte de l'article est certainement rédigé par René Coulon.

7. - AMOUROUX, Dominique. «La faculté des sciences de Rennes ». Dans AMOUROUX, Dominique. Louis Arretche. Paris: In Folio/éditions du Patrimoine, collection Carnets d'architecte, 2010, p. 48-51. «Pour maîtriser les fluctuations propres à chaque entité, Louis Arretche affecte un volume à chacune des composantes de son programme. Ce faisant, il allonge les halls et les parcours extérieurs, étire les circulations et développe les éléments de liaison extérieurs couverts. Rappelons que nous sommes à une époque où le coût du chauffage est faible et où les distances à parcourir à pied sont perçues comme une donnée normale de la vie quotidienne. » p. 50.

8. - «Faculté des sciences de Bordeaux ». L'Architecture française, nº 275-276, juillet-août 1965, p. 16.

9. - La ville de Bordeaux, à l'initiative de son architecte et urbaniste Jacques d'Welles, se dote en 1931 d'un comité d'architectes conseils, comité à vrai dire assez restreint et choisi puisqu'avant la guerre il a comme membres Jacques Debat-Ponsan et Roger-Henri Expert. Ce comité est notamment chargé d'examiner toutes les questions de constructions municipales. Certains projets sont même confiés directement à ses membres. Ainsi, en 1933, Expert est choisi pour étudier la reconstruction de la faculté des sciences.

10. - Jacques Carlu édifiera en 1965 l'École nationale supérieure des Arts et métiers, programme mitoyen de la faculté de Coulon. Il est également l'un des auteurs, à Bordeaux, de la cité de la Benauge sur la rive droite de la Garonne.

11. - «Jacques Carlu, rapport devant le Comité des architectes conseils de la ville de Bordeaux, séance du 5 mars 1953, archives municipales de Bordeaux, dossier du secrétariat général de la mairie ». Faculté des sciences de Talence, $10 \mathrm{~W} 105$.

12. - La bibliothèque des Sciences est due aux architectes Louis Sainsaulieu et Pierre Daurel. Elle est livrée en 1963.

13. - P. Carpentier, directeur général de l'équipement scolaire, universitaire et sportif, dans une préface au dossier « Constructions scolaires ». Techniques et architectures, n 3, mars 1960, p. 87.

14. - Cité par Carlu dans son rapport du 5 mars 1953. Livrée en 1957, la faculté des sciences de Dijon est l'œuvre du Dijonnais Robert Barade, architecte du département de la Côte-d'Or. Celui-ci représente un cas presque exceptionnel d'un architecte auteur de tout un domaine universitaire, Montmuzard, où sont transférés tous les locaux universitaires situés avant dans le centre de Dijon. Sur le domaine universitaire de Dijon voir: BEJEAN, Sophie. «L'Université de Bourgogne, 
un campus à l'œuvre... ». Dans POIRRIER, Philippe (dir.). Paysage des campus. Urbanisme, architecture et patrimoine. Dijon : Presses universitaires de Dijon, 2009, p. 126-147.

15. - Note du secrétaire général de mairie à Chaban-Delmas datée du 18 mars 1953, arch. mun. de Bordeaux, $10 \mathrm{~W} 105$.

16. - Lettre d'André Marie, ministre de l'Éducation nationale au maire de Bordeaux, 29 avril 1953, arch. mun. de Bordeaux, $10 \mathrm{~W} 105$.

17. - Le centre d'archives d'architecture du XXe siècle de la Cité de l'Architecture et du Patrimoine conserve dans le fonds de la Direction de l'architecture et de l'urbanisme un dossier sur René-André Coulon duquel sont issus les documents anciens reproduits dans cet article.

18. - La ville nouvelle de Mourenx est créée près de Pau dans les Pyrénées-Atlantiques pour loger les employés du gisement pétrolier de Lacq. Les études, confiées à René Coulon, J. Maneval et Philippe Douillet, débutent en 1957. La ville est bâtie en un temps record et achevée en 1961. Sur Mourenx et Lacq, voir : Techniques et architecture, $\mathrm{n}^{\circ}$ 6, octobre 1959.

19. - DEHAN, Philippe. «René-André Coulon (1908-1997)». L'Architecture d'aujourd'hui, n 310, avril 1997, p. 17.

20. - Les deux premiers bâtiments de la faculté de Jussieu construits en 1856, l'un avec une structure en béton et l'autre avec une structure métallique, mais recouverts des mêmes panneaux de briques enduites, ont été largement éclipsés de l'historiographie par le "grill» d'Édouard Albert. Sur ces deux bâtiments, on peut consulter: «Paris-Orsay. Faculté des sciences ». Techniques et architecture, $\mathrm{n}^{\circ} 3$, mars 1960, p. 120-121. Pour la construction de l'université de Jussieu, on peut consulter: SEITZ, Frédéric. L'architecture métallique au XXe siècle. Paris: Belin, p. 122. Sur leur postérité, on se rapportera à : HOTTIN, Christian. "Jussieu, l'inachevée ». Dans POIRRIER, Philippe (dir.). Paysage des campus. Urbanisme, architecture et patrimoine. Dijon : Presses universitaires de Dijon, 2009, p. 55-70.

21. - JULLIAN, René. Histoire de l'architecture en France de 1889 à nos jours. Un siècle de modernité. Paris : Philippe Sers, 1984, p. 235.

22. - DEHAN, Philippe. «René-André Coulon (1908-1997) ». L'Architecture d'aujourd'hui, n 310, avril 1997.

23. - SCHEIN, Ionel. «France construite ». Bauen + Wohnen, mai 1963, p. 86.

24. - «Jouy-en-Josas. École des Hautes études commerciales». Techniques et architecture, 1965, p. 119-122.

25. - Georges de Sonneville (1889-1978). Catalogue d'exposition, Bordeaux, Musée d'Aquitaine, 1990.

\section{RÉSUMÉS}

$\mathrm{Au}$ sortir de la seconde guerre mondiale, les facultés bordelaises étouffent dans leurs locaux du centre-ville édifiés sous la troisième République. Un ambitieux programme de transferts des établissements universitaires vers les banlieues de Pessac, Talence et Gradignan se met en place à partir des années 1950. L'architecte René-André Coulon (1908-1997) conçoit la faculté des sciences de Bordeaux, envisagée alors comme une véritable faculté "pour l'an 2000 ». Tout en disposant largement les différents bâtiments dans l'espace du campus, Coulon s'attache à leur donner un style original, tout empreint de rigueur et de rationalisme, mais qui montre également quelques écarts notables avec les normes régissant alors les constructions universitaires. Talence est la première réalisation universitaire de Coulon, qui travaillera par la suite sur des 
programmes fort différents, qu'il s'agisse du campus de HEC à Jouy-en-Josas ou de celui de la faculté des sciences de Paris-centre avec Édouard Albert.

INDEX

Mots-clés : René-André Coulon, Talence, Bordeaux, faculté des sciences de Bordeaux, campus, Édouard Albert, université

\section{AUTEUR}

\section{FRANCK DELORME}

Historien de l'architecture, attaché de conservation à la Cité de l'Architecture et du Patrimoine / centre d'archives d'architecture du XX ${ }^{\mathrm{e}}$ siècle fdelorme@citechaillot.fr 